Obstetrics

\title{
Spouse's social support in the postpartum period, predictors and its relationship with postpartum depression in a sample of Iranian primiparous women
}

\author{
Zahra Eslahi M.Sc ${ }^{1}$ | Nasim Bahrami ${ }^{2}$ | Kelly-Ann Allen ${ }^{3}$ | Zainab Alimoradi ${ }^{2}$
}

\begin{abstract}
${ }^{1}$ Students' Research Committee, School of Nursing \& Midwifery, Qazvin University of Medical Sciences, Qazvin, Iran

${ }^{2}$ Social Determinants of Health Research Center, Research Institute for Prevention of Non-Communicable Diseases, Qazvin University of Medical Sciences, Qazvin, Iran ${ }^{3}$ Faculty of Education, Monash University, Clayton, Vic, Australia
\end{abstract}

\section{Correspondence}

Nasim Bahrami and Zainab Alimoradi, Social Determinants of Health Research Center, Research Institute for Prevention of Non-Communicable Diseases, Qazvin University of Medical Sciences, Qazvin 34197-59811, Iran.

Email: n.bahrami@qums.

ac.ir;nbahrami87@gmail.com

Email: z.alimoradi@qums.ac.ir; zainabalimoradi.sbmu.ac.ir@gmail.com

Funding Information

This study was financially supported by the Vice-Chancellor (Research) of Qazvin University of Medical Sciences. The funding body had no role in the design of the study, collection, analysis, interpretation of data, and writing the manuscript

\begin{abstract}
Objective: To investigate the predictive factors of receiving spousal support in the postpartum period and its relationship with postpartum depression (PPD).

Methods: This cross-sectional study was conducted between January and May 2019 in 250 primiparous women to determine the predictors of spousal social support in the postpartum period. Three scales were used to collect data: The Demographic and Obstetric Checklist, the Postpartum Partner Support Scale, and the Edinburgh Postpartum Depression Scale.

Results: Multivariate regression showed that the employment status of the spouse and life satisfaction variables were predictive of whether social support was received from a spouse in the postpartum period. In total, the variables examined in this model explained $19 \%$ of the variance for a mother receiving spousal social support in the postpartum period. PPD and spousal social support had a moderately inverse and significant correlation ( $\beta=-0.39$ ).

Conclusion: Life satisfaction and employment of the spouse are important predictive variables for receiving social support of the spouse in the postpartum period. There is also a significant inverse relationship between PPD and spousal social support.
\end{abstract}

\section{KEYWORDS}

depression, postpartum, social support

\section{1 | INTRODUCTION}

Being a mother can be one of the most enjoyable events in a woman's life; however, motherhood can also be accompanied by increased physical and mental demands and stressors. ${ }^{1}$ The postpartum period begins immediately after the baby is born and lasts for up to 6 weeks after birth. The World Health Organization (WHO) describes this period as the most important stage in the lives of mothers and infants. ${ }^{2}$

The birth of a child can lead to a dynamic change in the family, which can be a source of physical and emotional vulnerability for all family members, but especially mothers. ${ }^{3}$ However, receiving social support has been found to be an important factor in reducing a mother's vulnerability and stress. ${ }^{4}$ Social support represents the emotional, psychological, or physical support provided from another person. Family members, friends, neighbors, co-workers, relatives, and clinicians are common sources of social support for mothers during the postpartum period. These individuals can provide psychological support to mothers as well as informational support. ${ }^{5}$ Family-based support from someone with an intimate and ongoing interpersonal relationship, such as a spouse, has been found to increase a mother's perceived parenting skills and self-confidence. Spousal support for mothers has also been reported to strengthen a mother's ability to adapt and deal with the new stressors after childbirth and to improve the strengthening of the mother-child relationship. ${ }^{4}$

Maternal fatigue, postpartum blues, depression, anxiety, and postpartum stress are conditions that require support, especially for 